\title{
Built-in Self-Test and Fault Diagnosis for Lab-on-Chip Using Digital Microfluidic Logic Gates*
}

\author{
Yang Zhao, Tao Xu and Krishnendu Chakrabarty \\ Department of Electrical and Computer Engineering \\ Duke University, Durham, NC 27708, USA
}

\begin{abstract}
Dependability is an important system attribute for microfluidic lab-on-chip. Structural test and functional test are needed to detect defects and malfunctions, respectively. Previously proposed techniques for reading test outcomes and for pulse-sequence analysis are cumbersome and error-prone. We present a built-in self-test (BIST) method for digital microfluidic lab-on-chip. This method utilizes digital microfluidic logic gates to implement the BIST architecture; AND, OR and NOT gates are used to compress test-outcome droplets into one droplet signature. This approach obviates the need for capacitive sensing test-outcome circuits for analysis. An efficient diagnosis method based on a microfluidic encoder is also proposed to locate a single defective electrode in a microfluidic array. Finally, reconfiguration is used during test and diagnosis to enable BIST with low area overhead.
\end{abstract}

\section{INTRODUCTION}

Microfluidics lab-on-chip technology has made great strides in recent years [1]. It has enabled on-chip immunoassays, clinical diagnosis, environmental toxicity monitoring, and highthroughput DNA sequencing. An especially promising technology platform is based on the principle of electrowettingon-dielectric. Discrete droplets of nanoliter volumes can be manipulated in a "digital" manner on a two-dimensional array of electrodes ("unit cells"). Hence this technology is referred to as "digital microfluidics" [2].

Another application of microfluidics lies in the use of droplets for implementing logic gates [6]. Microfluidic logic gates can be implemented in various ways, such as electrochemical reactions [16], relative resistance [14], bubbles in electronic channels [8]. However, a drawback of these methods is that they assign different interpretations to inputs and outputs, which makes cascading of gates difficult. Digital microfluidics provides a promising alternative technique for on-chip logic functionality, and for integrating sensing and computing.

As more bioassays are mapped on a microfluidic platform for concurrent execution, system complexity and integration levels are expected to increase steadily. A prototype has been developed for gene sequencing through synthesis [1], which

*This work was supported in part by the National Science Foundation under grant CCF-0541055. targets the simultaneous execution of 106 fluidic operations and the processing of billions of droplets. Other lab-onchip systems are being designed for protein crystallization, which requires the concurrent execution of hundreds of operations [19]. A commercially available droplet-based (using dielectrophoresis) lab-on-chip embeds more than 600,000 20 $\mu \mathrm{m}$ by $20 \mu \mathrm{m}$ electrodes with integrated optical detectors [10]. Recent years have therefore seen growing interest in designautomation and test techniques for the digital microfluidic platform [4], [9], [12], [13], [15]. Test techniques for other microfluidic platforms have also been developed [5].

An increase in the density and area of microfluidics-based lab-on-chip will lead to high defect densities, thereby reducing yield. Dependability is an important system attribute for labon-chip, especially for safety-critical applications such as point-of care diagnostics, health assessment and screening for infectious diseases. Some manufacturing defects may produce errors during field operation. Therefore, structural test is needed to detect these defects. For instance, a single defective electrode can be detected/located via the traversal of a test droplet. In [17], a parallel scan-like testing methodology has been proposed for digital microfluidic devices. A diagnosis method based on test outcomes has also been proposed to locate both single and multiple defect sites.

Many fluidic operations must be repeatedly executed with high precision using a group of unit cells in compact microfluidic arrays. Structural test methods, which use test droplets to traverse the target array, are not sufficient to ensure that these fluidic operations can be reliably performed on the array. For instance, some unit cells may function correctly during droplet transportation, but they might malfunction during droplet dispensing from reservoirs. Likewise, a group of cells, which can be reliably utilized to operate as a mixer, may malfunction when they are used for droplet splitting. Therefore, it is important to carry out functional testing to verify the functionality of the underlying microfluidic platform. In [18], several techniques are proposed for the functional testing of droplet-based microfluidic biochips. These techniques address fundamental biochip operations such as droplet dispensing, transportation, mixing, splitting, and capacitive sensing.

Previous test methods for digital microfluidic platforms use capacitive sensing circuits to read and analyze test outcomes [17], [18]. After reading the test-outcome droplets in a consecutive manner, the capacitive sensing circuit generates a pulse-sequence corresponding to the detection of these 


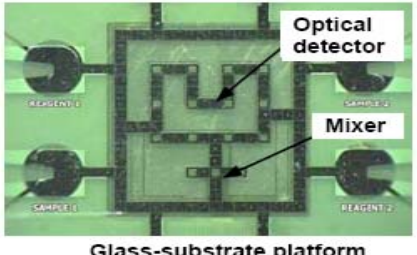

Glass-substrate platform

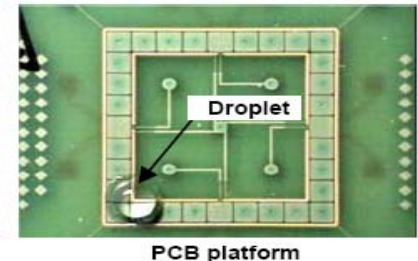

PCB platform
Fig. 1. Fabricated digital microfluidic arrays [18].

droplets. This approach requires an additional step to analyze the pulse sequence to determine whether the microfluidic array under-test is defective. The reading of test outcomes and the analysis of pulse sequences increase test time; the latter procedure is especially prone to errors arising from inaccuracies in sensor calibration. The complexity of the capacitivesensing circuit and the need for pulse-sequence analysis make previously proposed testing methods less practical, especially for field operation.

In this paper, we propose a built-in self-test (BIST) method for digital microfluidic lab-on-chip. This method utilizes microfluidic logic gates to implement the "compactor" in a BIST architecture. Using the principle of electrowetting-ondielectric, we implement AND, OR and NOT gates through basic droplet-handling operations such as transportation, merging, and splitting. The same input-output interpretation enables the cascading of gates for the implementation of additional logic functions. The microfluidic compactor can compress the test-outcome droplets into one droplet in a very short amount of time, and the droplet can be detected using a simple photodiode detector, thereby avoiding the need for a capacitivesensing circuit and complicated pulse-sequence analysis. We also propose an efficient diagnosis method based on a "microfluidic encoder" to locate a single defective electrode in a microfluidic array. Reconfiguration is used to achieve low area overhead.

The rest of the paper is organized as follows. Section 2 provides an overview of the digital microfluidic platform. In Section 3, we present digital microfluidic AND, OR and NOT gates. In Section 4 and 5, we use these logic gates for parallel scan-like testing and functional testing. A compactor is proposed to compress the test-outcome droplets. In Section 6, we describe a diagnosis method based on the reconfiguration of microfluidic logic gates. Finally, conclusions are drawn in Section 7.

\section{Digital Microfluidic Platform}

In digital microfluidics, droplets of nanoliter volumes are manipulated on a two-dimensional electrode array [1]. A unit cell in the array includes a pair of electrodes that acts as two parallel plates. The bottom plate contains a patterned array of individually controlled electrodes, and the top plate is coated with a continuous ground electrode. A droplet rests on a hydrophobic surface over an electrode, as shown in Fig. 1. Coplanar microfluidic arrays (without a top plate) have also been fabricated [7].

Droplets are moved by applying a control voltage to a unit cell adjacent to the droplet and, at the same time, deactivating

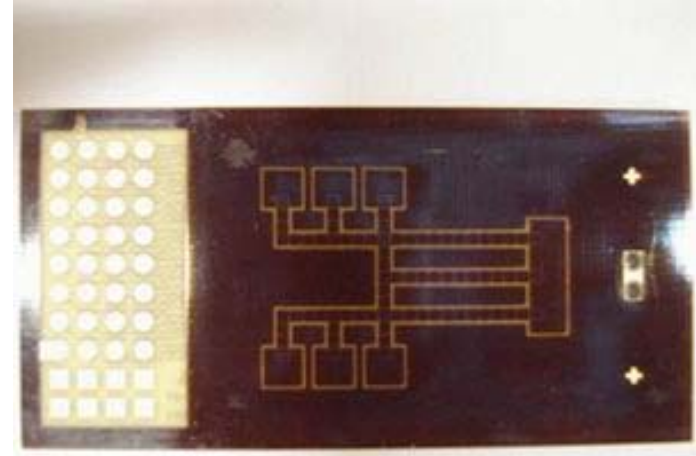

Fig. 2. Fabricated lab-on-chip used for experiments.

the cell under the droplet. This electronic method of wettability control creates interfacial tension gradients that move the droplets to the charged electrode. Fluid-handling operations such as droplet merging, splitting, mixing, and dispensing can be executed in a similar manner. Droplet routes and operation schedules are programmed into a microcontroller that drives the electrodes.

\section{Microfluidic Logic Gates}

In the digital microfluidic platform, droplets of unit volume (1x) or larger can be easily moved [1]. A droplet of $0.5 x$ volume is not large enough to have sufficient overlap with an adjacent electrode; hence it cannot be moved with a nominal actuation voltage. It has been verified experimentally that the times required for dispensing one droplet, splitting a droplet into two, merging two droplets into one, and transporting a droplet to an adjacent electrode are nearly identical. This duration is defined as one time frame (clock cycle).

The definitions of logic values ' 0 ' or ' 1 ' are as follows: the presence of a droplet of $1 \mathrm{x}$ volume at an input or output port indicates a logic value of ' 1 '. The absence of a droplet at an input or output port indicates the logic value ' 0 '. The same interpretations at inputs and outputs enable the output of one gate to be fed as an input to another gate, thus logic gates can be easily cascaded.

In this section, we present the schematics and actuation voltage sequences for various microfluidic logic gates. The cycle-by-cycle operation of gates is also presented. To experimentally verify the functionality of these gates, we configure them on a fabricated lab-on-chip, then activate the corresponding electrodes to perform the cycle-by-cycle operations.

A typical experimental setup is as follows. The chip-undertest is mounted on a custom-assembled platform. We use a custom-made electronic unit to independently control the voltages of each control electrode in the array by switching them between ground and a DC actuation voltage. In our experiments, the actuation voltage was set to $50 \mathrm{~V}$. The chip-under-test is a PCB microfluidic prototype for protein crystallization, as shown in Fig. 2. 


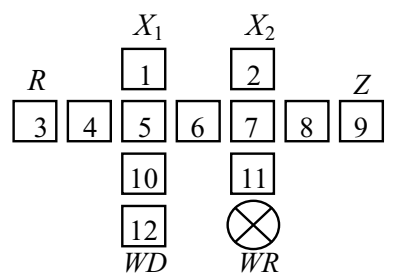

(a) OR gate: $Z=X_{1}+X_{2}$

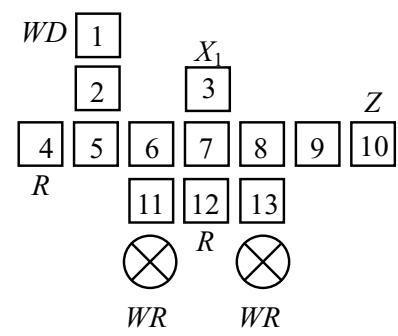

(c) NOT gate: $Z=\overline{X_{1}}$

Fig. 3. Schematics of microfluidic logic gates.

TABLE I

ACtUATION-VOLTAGE SEQUENCE FOR THE OR GATE.

\begin{tabular}{|c|c|c|c|c|c|c|c|c|c|c|c|c|}
\hline Clock & \multicolumn{12}{|c|}{ Electrode No. } \\
\hline cycle & 1 & 2 & 3 & 4 & 5 & 6 & 7 & 8 & 9 & 10 & 11 & 12 \\
\hline 0 & 1 & 1 & 1 & 0 & 0 & $\mathrm{~F}$ & 0 & $F$ & $\mathrm{~F}$ & 0 & $\mathrm{~F}$ & 1 \\
\hline 1 & 0 & 0 & 1 & 0 & 1 & 0 & 1 & 0 & $\mathrm{~F}$ & 0 & 0 & 1 \\
\hline 2 & 0 & 0 & 1 & 0 & 0 & 1 & 0 & 0 & $\mathrm{~F}$ & 0 & 0 & 1 \\
\hline 3 & 0 & 0 & 1 & 0 & 1 & 0 & 1 & 0 & $\mathrm{~F}$ & 0 & 0 & 1 \\
\hline 4 & 0 & 0 & 0 & 1 & 0 & 0 & 0 & 0 & $\mathrm{~F}$ & 0 & 1 & 1 \\
\hline 5 & 0 & $\mathrm{~F}$ & 0 & 0 & 1 & 0 & 0 & $\mathrm{~F}$ & $\mathrm{~F}$ & 0 & 0 & 1 \\
\hline 6 & 0 & $\mathrm{~F}$ & $\mathrm{~F}$ & 0 & 0 & 1 & 0 & $\mathrm{~F}$ & $\mathrm{~F}$ & 0 & $\mathrm{~F}$ & 1 \\
\hline 7 & $\mathrm{~F}$ & 0 & $\mathrm{~F}$ & $\mathrm{~F}$ & 0 & 0 & 1 & 0 & $\mathrm{~F}$ & 0 & 0 & 1 \\
\hline 8 & $\mathrm{~F}$ & 0 & $\mathrm{~F}$ & $\mathrm{~F}$ & 0 & 1 & 0 & 1 & 0 & 0 & 0 & 1 \\
\hline 9 & $\mathrm{~F}$ & $\mathrm{~F}$ & $\mathrm{~F}$ & $\mathrm{~F}$ & 0 & 1 & 0 & 0 & 1 & 0 & $\mathrm{~F}$ & 1 \\
\hline
\end{tabular}

\subsection{Microfluidic OR Gate}

Fig. 3 shows schematics for the 2-input OR, 2-input AND, NOT, and 2-input multiplexer gates. The OR gate in Fig. 3(a) incorporates a waste reservoir $(W R)$ and twelve indexed electrodes. Electrode 1 and Electrode 2 are the two input ports $X_{1}$ and $X_{2}$; Electrode 3 is the reference port $(R)$, from which one reference droplet is injected into the OR gate. Electrode 9 is the output port $(Z)$ where a detector can be placed to determine the output logic value of the OR gate. Such detections to indicate the presence or absence of a droplet can be easily implemented using photo-diodes [11]. Electrode 12 is the washing port $(W D)$, from which a washing droplet is injected after the logic operation to collect the residual droplets and move them to the waste reservoir.

The sequence of control voltages applied to each electrode is shown in Table I. A ' 1 ' (' 0 ') entry in the table indicates a high (low) voltage to the corresponding electrode in that clock cycle. An ' $F$ ' entry indicates a floating signal, i.e., the corresponding electrode is not required to be either high or low. The sequence of control voltages is independent of the

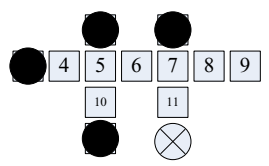

(a) $t=0$, two $1 \mathrm{x}$ droplets stay at input ports.

(d) $t=3$, a $2 \mathrm{x}$ droplet is split into two $1 \mathrm{x}$ droplets.

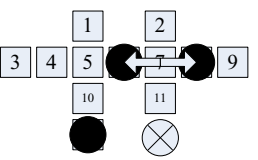

(g) $t=8$, a $2 \mathrm{x}$ droplet is split into two $1 \mathrm{x}$ droplets.

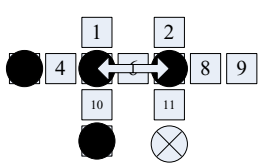

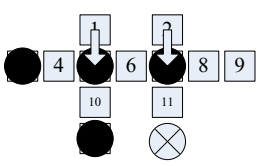

(b) $t=1$, two $1 \mathrm{x}$ droplets move downwards.

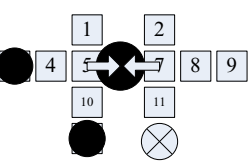

(c) $t=2$, two $1 \mathrm{x}$ droplets are merged into a $2 \mathrm{x}$ droplet.

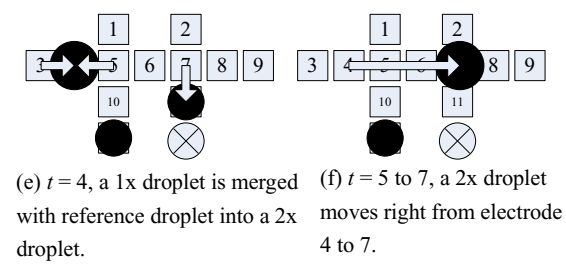

Fig. 4. Operation of the OR gate for input 11 .

input logic values.

Fig. 4 describes the cycle-by-cycle operation of the OR gate for $X_{1} X_{2}=11$. At clock cycle 0 , two $1 \mathrm{x}$ droplets stay at two input ports (Electrode 1 and 2). At clock cycle 9, there is a 1x droplet on electrode 9, hence the output value is 1 .

The delay of the OR gate is 9 clock cycles, independent of the inputs. At the beginning of clock cycle 10, the droplet on the washing port (Electrode 12) is routed to merge with the residual droplets, and the result is transported to the waste reservoir. After this washing process, no droplet is left on the electrodes, and this gate is clean for the next operation.

To experimentally verify the OR gate, we configured it on the fabricated lab-on-chip, then activated the corresponding electrodes to perform on-chip cycle-by-cycle operations in the laboratory. Fig. 5 shows the operation of the gate for $X_{1} X_{2}$ $=11$. When $t=0$, two $1 \mathrm{x}$ droplets stay on the electrodes representing two inputs, while one $1 \mathrm{x}$ droplet stays on the electrode representing the reference port. Operations from $t=$ 1 to 10 are the same as that in Fig. 4. Note that the splitting step in the experiment occupies five electrodes and lasts for three clock cycles, as shown from $t=2$ to 4 . This is because we want to ensure even and thorough splitting, to acquire two split droplets with equal volume. At $t=11$, there is one $1 \mathrm{x}$ droplet on the electrode representing the output. Experimental results demonstrate the feasibility of the OR gate for different input values.

\subsection{Microfluidic AND Gate}

Fig. 3(b) illustrates the schematic of a 2-input microfluidic AND gate. The AND gate in Fig. 3(b) incorporates a waste reservoir $(W R)$ and nine indexed electrodes. Electrode 1 and Electrode 2 are the two input ports $X_{1}$ and $X_{2}$; Electrode 7 is the output port $(Z)$. Electrode 9 is the washing port $(W D)$.

The sequence of control voltages applied to each electrode is shown in Table II. Fig. 6 describes the cycle-by-cycle 


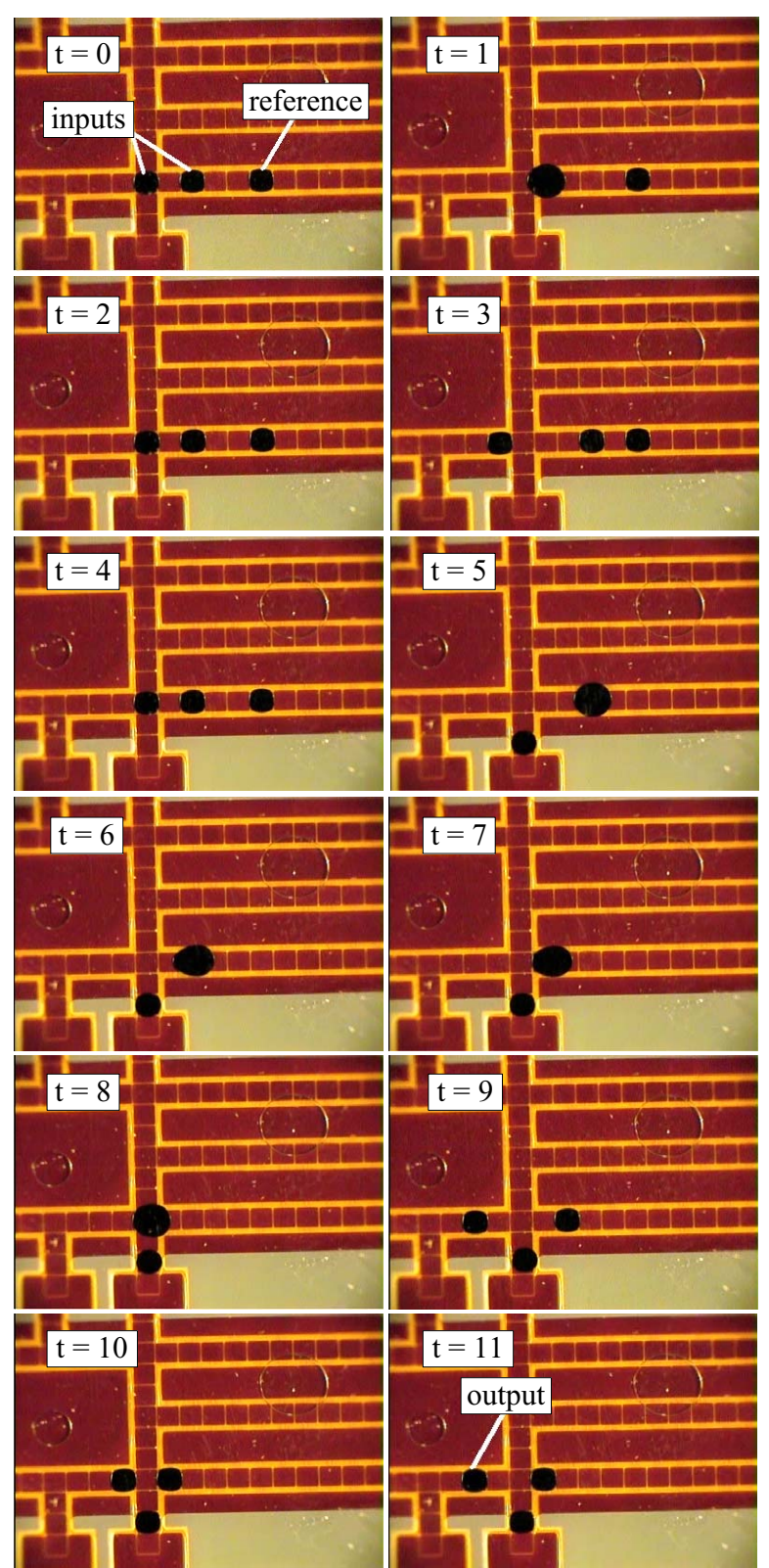

Fig. 5. On-chip cycle-by-cycle operation for the OR gate with input 11 .

TABLE II

ACTUATION-VOLTAGE SEQUENCE FOR THE AND GATE.

\begin{tabular}{||c|c|c|c|c|c|c|c|c|c||}
\hline Clock & \multicolumn{10}{|c||}{ Electrode No. } \\
\cline { 2 - 11 } & 1 & 2 & 3 & 4 & 5 & 6 & 7 & 8 & 9 \\
\hline 0 & 1 & 1 & 0 & $\mathrm{~F}$ & $\mathrm{~F}$ & $\mathrm{~F}$ & $\mathrm{~F}$ & 0 & 1 \\
\hline 1 & 0 & 0 & 1 & 0 & $\mathrm{~F}$ & $\mathrm{~F}$ & $\mathrm{~F}$ & 0 & 1 \\
\hline 2 & 0 & 1 & 0 & 1 & 0 & $\mathrm{~F}$ & $\mathrm{~F}$ & 0 & 1 \\
\hline 3 & $\mathrm{~F}$ & 0 & 0 & 0 & 1 & 0 & $\mathrm{~F}$ & 0 & 1 \\
\hline 4 & $\mathrm{~F}$ & $\mathrm{~F}$ & $\mathrm{~F}$ & 0 & 0 & 1 & 0 & 0 & 1 \\
\hline 5 & $\mathrm{~F}$ & $\mathrm{~F}$ & $\mathrm{~F}$ & $\mathrm{~F}$ & 0 & 0 & 1 & 0 & 1 \\
\hline
\end{tabular}

operation of the AND gate for $X_{1} X_{2}=11$. At clock cycle 0 , two 1x droplets stay at two input ports (Electrode 1 and 2). At clock cycle 5, there is a $1 \mathrm{x}$ droplet on electrode 9, showing that the output value of this AND gate is 1 .

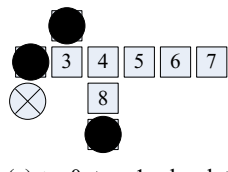

(a) $t=0$, two $1 \mathrm{x}$ droplets stay at the input ports.

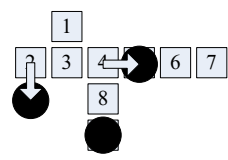

(d) $t=3$, a $1 \mathrm{x}$ droplet 4 to 5 . moves right from electrode

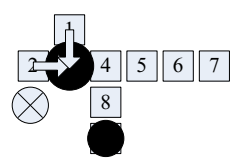

(b) $t=1$, two $1 \mathrm{x}$ droplets are merged into a $2 \mathrm{x}$ droplet.

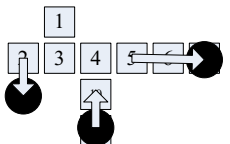

(e) $t=4$ to 5 , a $1 \mathrm{x}$ droplet moves right from electrode 5 to 7 . While the washing droplet moves upwards.
Fig. 6. Operation of the AND gate with input 11.

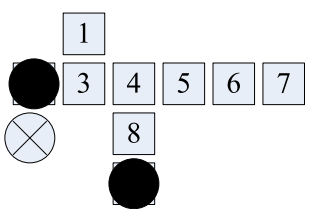

(a) $t=0$, one $1 \mathrm{x}$ droplet stays at the input port 1 .

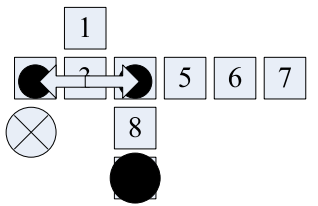

(c) $t=2$, a $1 \mathrm{x}$ droplet is split into two $0.5 \mathrm{x}$ droplets.

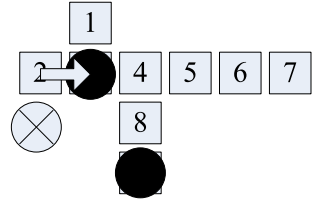

(b) $t=1$, one $1 \mathrm{x}$ droplet moves right from electrode 2 to 3 .

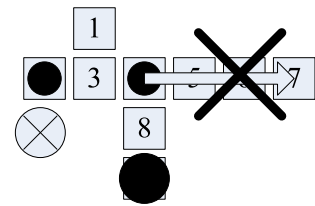

(d) $t=3$ to $5,0.5 \mathrm{x}$ droplet on electrode 4 cannot be moved to electrode 7 .
Fig. 7. Operation of the AND gate with input 10.

Fig. 7 illustrates the cycle-by-cycle operation of the AND gate for $X_{1} X_{2}=01$. At clock cycle 0 , one $1 \mathrm{x}$ droplet stays at the second input port (Electrode 2), while there is no droplet on the first input port (Electrode 1). At clock cycle 2, the $1 \mathrm{x}$ droplet on electrode 3 is split into two $0.5 \mathrm{x}$ droplets. The $0.5 \mathrm{x}$ droplet cannot be moved even if the adjacent electrode is activated at clock cycle 3 . Therefore, at clock cycle 5, there is no droplet on electrode 7, showing that the output value of this AND gate is 0 . Due to symmetry, $X_{1} X_{2}=10$ yields the same output value.

The delay of the AND gate is 5 clock cycles, independent of the inputs. At the beginning of clock cycle 6 , the droplet on the washing port (Electrode 9) is routed into the AND gate to clean the residuals and transport them to the waste reservoir.

\subsection{Microfluidic NOT Gate}

Fig. 3(c) shows a microfluidic inverter. The inverter incorporates two waste reservoirs $(W R)$ and thirteen indexed electrodes. Electrode 3 is the input port $X_{1}$; Electrode 10 is the output port $(Z)$; Electrode 4 and electrode 12 are two reference ports $(R)$, from each of which one reference droplet 


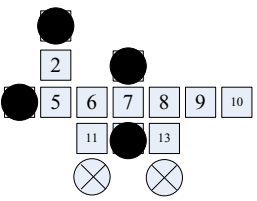

(a) $t=0$, one $1 \mathrm{x}$ droplet stays at the input port 1 .

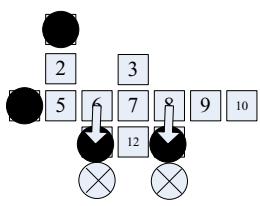
move downwards to waste reservoirs.

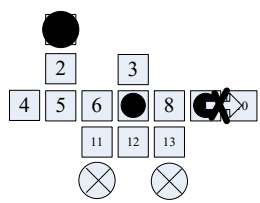

(g) $t=9,0.5 \mathrm{x}$ droplet on

electrode 9 cannot be moved to electrode 10 (d) $t=3$, two $1 \mathrm{x}$ droplets

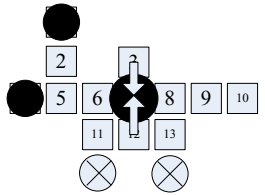

(b) $t=1$, input droplet is merged with reference droplet into a $2 \mathrm{x}$ droplet.

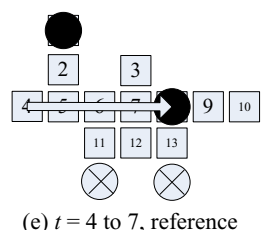

droplet moves right from electrode 4 to 8 .

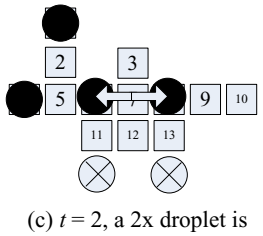

split into two $1 \mathrm{x}$ droplets.

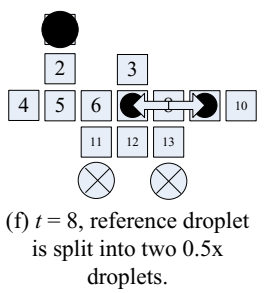
droplets.
Fig. 8. Operation of the inverter with input 1.

is injected into the inverter; Electrode 1 is the washing port $(W D)$.

Fig. 8 describes the cycle-by-cycle operation of the inverter for $X_{1}=1$. At clock cycle 0 , one $1 \mathrm{x}$ droplet stays at the input port (Electrode 3). At clock cycle 9, no droplet shows on electrode 10, indicating that the output value of this inverter is 0 . The delay of the inverter is 9 clock cycles.

\section{Application to Parallel Scan-like TEST}

In [17], a cost-effective testing methodology, referred to as "parallel scan-like test", has been proposed for fault detection and diagnosis for droplet-based microfluidic devices. All the defects listed in [17], e.g., dielectric breakdown, irreversible charge concentration on an electrode, droplet electrolysis, and metal connection between two adjacent electrodes, can be detected by manipulating test droplets to traverse the candidate faulty electrodes. In order to detect defects involving single unit cells and defects involving two cells, e.g., shorts between two adjacent electrodes, four iterations of test applications are needed to test the microfluidic array: two iterations for the vertically-connected pairs and two iterations to traverse all the horizontal connections. In each iteration, a test droplet is moved from its start electrode (referred to as pseudosource) along the row/column-under-test to its end electrode (referred to as pseudo-sink). Test droplets are routed in parallel along the corresponding rows/columns-under-test. After each iteration, to evaluate the test results, the test-outcome droplets are routed serially from "pseudo-sinks" to a capacitive-sensing circuit connected to the electrode for the sink reservoir. The

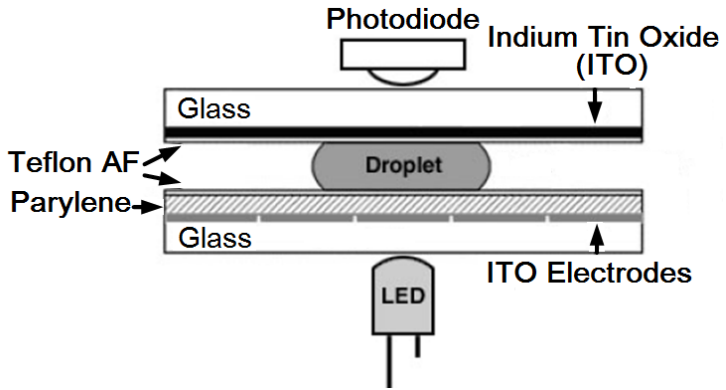

Fig. 9. Illustration of the use of the photo-diode detector [11].

capacitive-sensing circuit can produce a pulse-sequence corresponding to the detection of multiple test droplets.

After the test-outcome droplets are read serially, the capacitive-sensing circuit generates a pulse sequence corresponding to the detection of these droplets. An additional evaluation step is required to analyze these pulse-sequences to determine whether the microfluidic array under test has a defect. For example, if a row/column of an array is faulty, there is no generated pulse in the corresponding position of the pulse sequence. The complexity of the capacitive-sensing circuit and the need to analyze complex pulse-sequence is a major drawback of [17]. Moreover, there is a need to calibrate the pulse-analysis system and errors are likely due to the lack of a "noise margin".

To solve the above problem, we propose a microfluidic compactor to compress multiple test-outcome droplets into one test-outcome droplet, which can be easily read and detected by a simple detector composed of a photo-diode and LED, as shown in Fig. 9 [11]. The microfluidic compactor consists of a tree of microfluidic 2-input AND gates. The input ports of the AND gates in the first layer are connected one-by-one to the pseudo-sinks of the parallel scan-like row/column test, while the output ports of these AND gates are connected one-byone to the input ports of AND gates in the second layer. The output of the single AND gate in the last layer is connected to the photo-diode detector located at the sink of the microfluidic array.

Fig. 10 illustrates the schematic of the microfluidic compactor for parallel scan-like test of odd rows/columns in a $16 \times 16$ microfluidic array. The electrodes represent the last row/column where the pseudo-sinks are located. The compactor consists of three layers of microfluidic 2-input AND gates. The output of the AND gate in the third layer is connected to the photo-diode detector located in the sink reservoir of the microfluidic array.

In Fig. 10(a), after the parallel scan-like test for odd rows/columns, each of the odd pseudo-sinks has one $1 \mathrm{x}$ droplet on it, indicating that there is no defect in any odd rows/columns. The arrows show the direction of droplet routing through the AND gates. The number at each arrow indicates the logic value of the input or output, according to the definitions for logic values in microfluidic systems. The input to each AND gate in the first layer is ' 1 ', so its output is ' 1 '. A $1 \mathrm{x}$ droplet finally appears on the output port (electrode) 


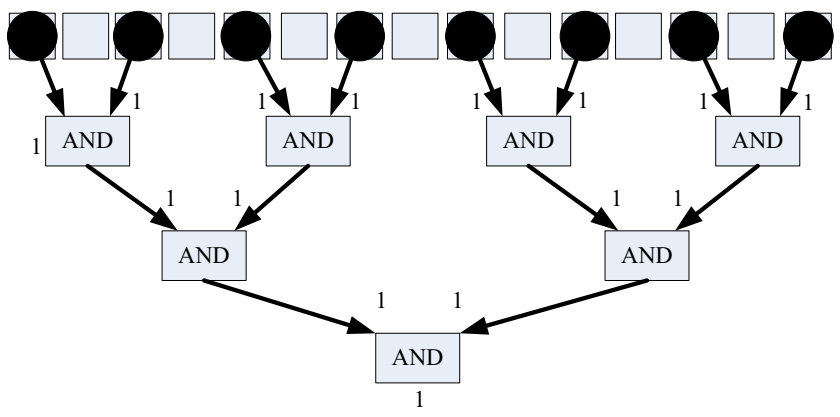

(a)

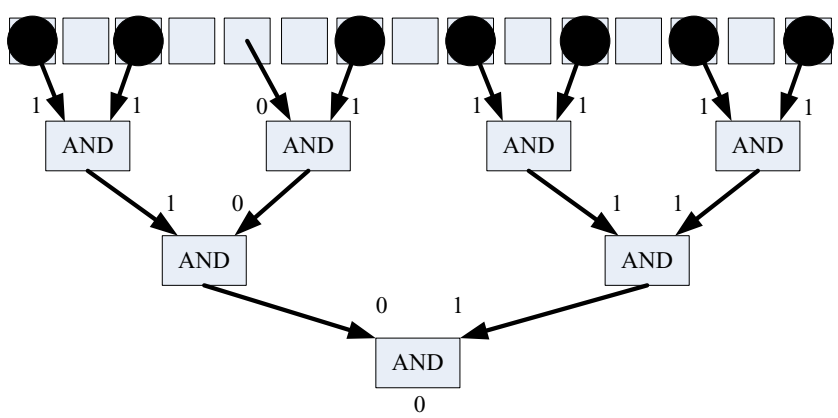

(b)

Fig. 10. Schematic of the microfluidic compactor for parallel scan-like testing: (a) compactor output $=$ ' 1 '; (b) compactor output $=$ ' 0 '.

of the microfluidic compactor, indicating that the output value of the compactor is ' 1 '. Instead of routing all the droplets in odd pseudo-sinks one-by-one to the capacitive-sensing circuit and analyzing the resulting pulse-sequence, we use the photodiode detector to check for the presence of the $1 \mathrm{x}$ droplet on the output of the compactor. We conclude that there is no defect in the odd rows/columns of the microfluidic array.

In Fig. 10(b), there is no droplet on the fifth electrode, indicating that the corresponding row/column has a defect. Therefore, the left input of the second AND gate in the first layer is ' 0 ', while other input values are ' 1 '. The output of the AND gate with a ' 0 ' input value is ' 0 '. No droplet appears on the output port (electrode) of the microfluidic compactor, therefore the output value of the compactor is ' 0 '. Since no droplet is detected by the photo-diode detector, we conclude that there is a defect in the odd rows/columns under test.

Fig. 11 shows the placement of the microfluidic compactor whose schematic is shown in Fig. 10 . The $16 \times 16$ microfluidic array is also shown. The last three rows of electrodes are used to construct the microfluidic compactor. Note that we use a simplified design of the AND gate, which consists of only four electrodes. In order to reduce the size of AND gate, the washing port and waste reservoir are not included in the AND gate. The microfluidic compactor consists of 3 layers of 2-input AND gates. Only one washing port and one waste reservoir are needed for the compactor. After the compaction of the test-outcome droplets, a droplet from the washing port is routed through each of the electrodes in the compactor, merged with the waste droplets, and routed to the waste reservoir.

The microfluidic array has only one source reservoir and

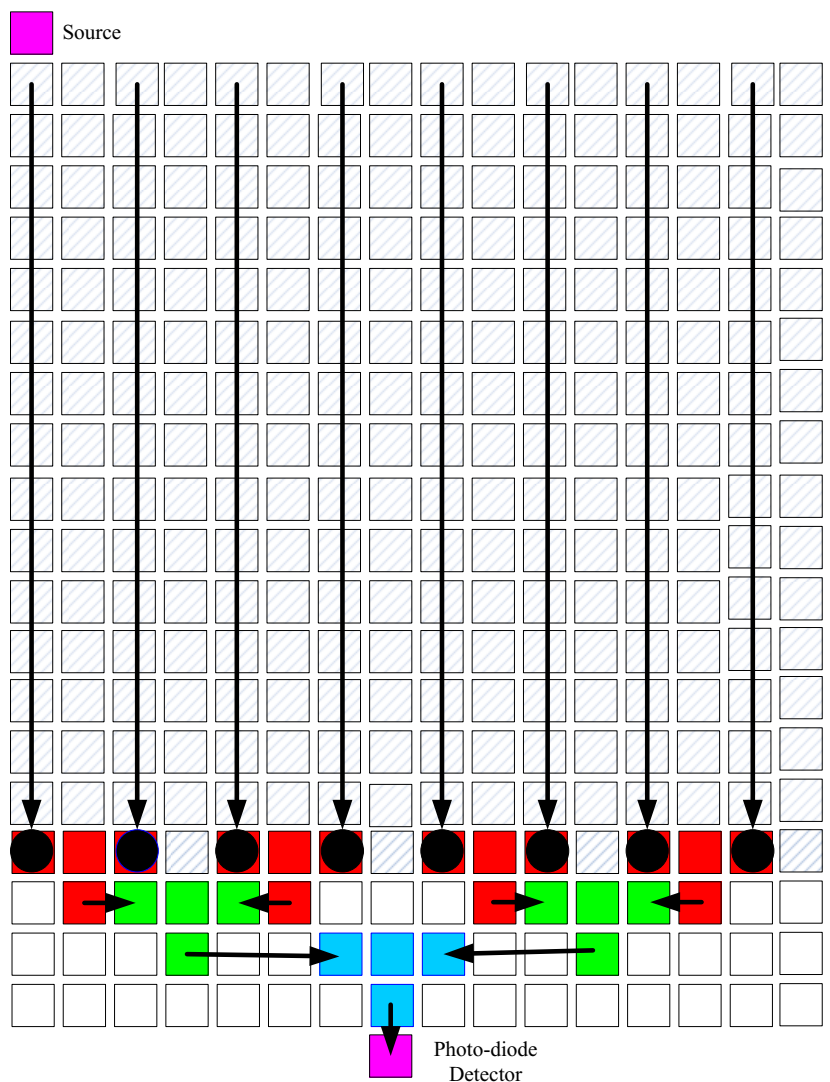

Fig. 11. Placement of the microfluidic compactor for parallel scan-like testing.

one sink reservoir where the photo-diode detector is located. This simplifies chip packaging and reduces fabrication cost. Dispensed from the single source, test droplets are aligned one-by-one and routed in sequence, as components in an assembly line, along the periphery nodes to their pseudosources in the first row of all the odd columns. Starting from these pseudo-sources, the test droplets are routed in parallel to the nodes at the other end of the corresponding odd columns. Finally, these test outcome droplets on the pseudosinks are compressed by the microfluidic compactor into one test-outcome droplet. As in Fig. 10, the arrows in Fig. 11 show the direction of the droplet flow. The output port of the compactor is directly connected to the photo-diode detector.

For the parallel scan-like test for even rows/columns of $16 \times 16$ microfluidic array, the microfluidic compactor is simply reconfigured by shifting it one column. The complete parallel scan-like test procedure with microfluidic compactor reconfiguration is shown in Fig. 12.

The proposed compactor guarantees zero aliasing, i.e., it does not mask any error in the microfluidic array. Complete coverage is ensured for all faults affecting a single unit cell as well as most faults that affect multiple unit cells. As discussed in [17], certain defect sites can be rendered untestable, and some unit cells can be incorrectly diagnosed as being defective.

For parallel scan-like test without a compactor, an $N \times$ $N$ microfluidic array needs $N$ clock cycles to route all test- 
1) Step 1. Peripheral Test: A test droplet is dispensed from the source. It is routed to traverse all the peripheral electrodes, and the droplet finally returns to the sink;

2) Step 2. Column Test:

i) 2(a): Parallel scan-like test of odd columns, where the test outcome droplets are compressed using microfluidic compactor, then the output of the compactor is read by the photo-diode detector;

ii) 2(b): Parallel scan-like test of even columns, where the microfluidic compactor is reconfigured with one column shift-right from odd columns.

3) Step 3. Row Test: Repeat parallel scan-like test for both the odd and even rows to detect defects. The microfluidic compactor is reconfigured with one row shift during the two iterations.

Fig. 12. Parallel scan-like test procedure with reconfiguration of microfluidic compactor.

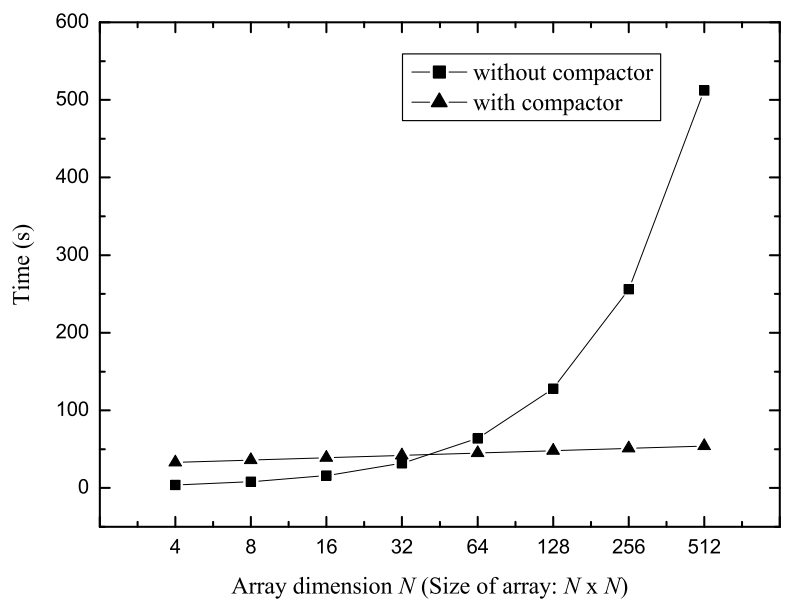

Fig. 13. Comparison of result-evaluation time with and without microfluidic compactor.

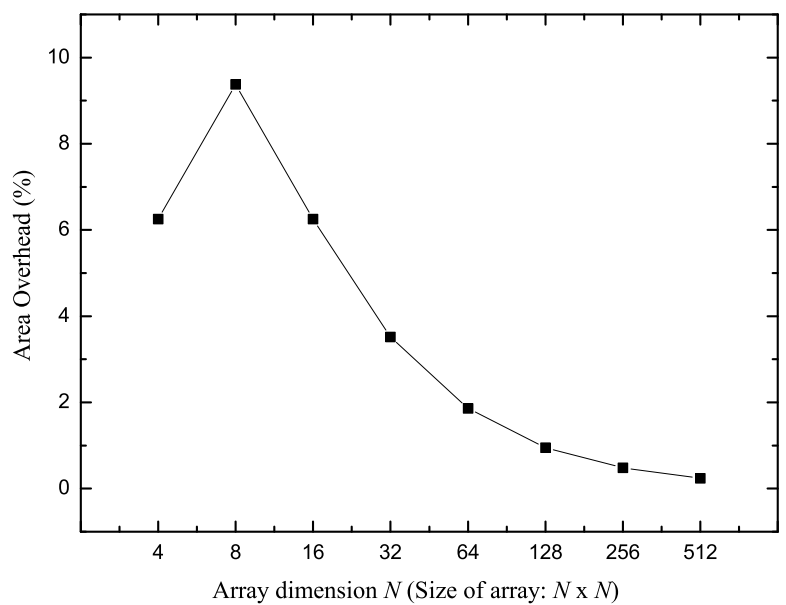

Fig. 14. Area overhead of the microfluidic compactor for parallel scan-like test.

outcome droplets consecutively to the sink node connected with the capacitive-sensing circuit. The time needed for capacitive-sensing is negligible [3]. For parallel scan-like test with a compactor, we need $3 \times\left(\log _{2} N-1\right)$ clock cycles to compress the test-outcome droplets to one droplet. The detection of the droplet at the compactor output takes 30 seconds using the photodiode detector [11]. This duration is comparable to the compaction time, therefore it must be taken into account when calculating the total time-cost for resultevaluation. The comparison of the result-evaluation time for the two methods, assuming a typical clock frequency of $1 \mathrm{~Hz}$, is shown in Fig. 13.

For an $N \times N$ microfluidic array, we need $\left(5 \times 2^{\log _{2} N-2}\right.$ - 4) electrodes to construct the compactor. For example, for $N=16$, we need $\left(5 \times 2^{\log _{2} 16-2}-4\right)=16$ electrodes. The area overhead of the compactor is shown in Fig. 14. Since each AND gate in the first layer of compactor utilizes three electrodes of the last row of the $N \times N$ microfluidic array under test, the area overhead for $N=4$ is less than that for $N=8$.

\section{Application to Functional Testing}

Functional testing for digital microfluidics was first introduced in [18]. It targets fluidic operations such as droplet dispensing, droplet transportation, mixing, and splitting. The proposed method allow functional testing using parallel droplet pathways. The functional test for the droplet-mixing operation is equivalent to the testing of the merging and routing operations within the target cell cluster. A mixing test can be reduced to a droplet merging test, which checks a series of three adjacent electrodes to determine whether two droplets can be merged on them. The fluidic splitting operation involves three adjacent electrodes, and can be viewed as the reverse of droplet merging. Therefore, splitting test can be carried out by applying the merging test methods in a reverse manner.

These two tests can be combined into a unified test application procedure. The key idea is to carry out mixing and splitting test for all the electrodes in a row/column concurrently. First, we carry out the horizontal splitting test for all the even electrodes in a row concurrently. The split droplets get merged at the odd electrodes, therefore the merging test is done at the same time. Second, by carrying out the splitting test for all the odd electrodes in a row concurrently, we can easily complete the horizontal merging test for all the even electrodes. Thus we can carry out all the horizontal tests (merging and splitting) in one row using only two manipulation steps. The testoutcome droplets on the electrodes after two manipulation steps are routed to the capacitive-sensing circuit. Similarly, all the vertical tests in one column can be completed in two manipulation steps.

Only one capacitive-sensing circuit is used in [18] to reduce hardware cost. Moreover, in order to minimize the number of droplet manipulations, test results are read out after splittingand-merging are carried out, as shown in Section 4. Therefore, a complicated test-result interpretation scheme is required. The complexity of the capacitive-sensing circuit and the testresult interpretation scheme make the functional test method impractical for field operation. 


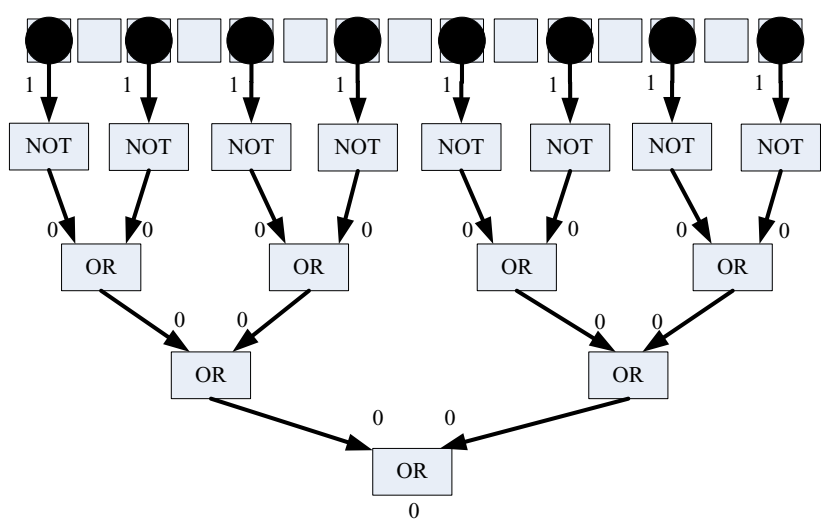

(a)

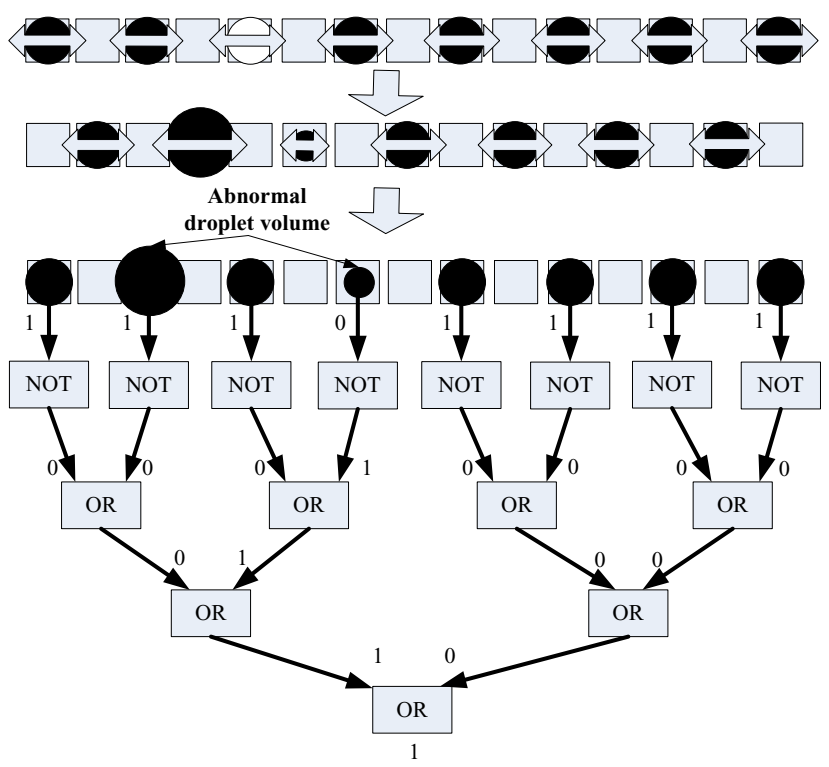

(b)

Fig. 15. Schematic of the microfluidic compactor for functional testing.

To address the above problem, a microfluidic compactor is used here to compress multiple test-outcome droplets into only one test-outcome droplet. This test-outcome droplet can be easily detected by a photo-diode detector. Suppose that during mixing/splitting test, a droplet undergoes an unbalanced split. Since all other droplets are split evenly, this malfunction results in a pair of test droplets of abnormal volume, one bigger and the other smaller. The bigger droplet (volume larger than $1 \mathrm{x}$ ) at the input ports of the AND gate is propagated to the output port, and it may lead to a malfunction of the AND gate. However, the bigger droplet does not affect the functionality of the NOT gate. Therefore, instead of AND gates, we use the combination of NOT gates and OR gates to construct the microfluidic compactor for functional testing.

The compactor consists of one layer of NOT gates and several layers of 2-input OR gates; see Fig. 15. The first layer consists of NOT gates, and the input port of each NOT gate is connected to each of the electrodes on which the droplets stay after the mixing and splitting test for one row/column. Other layers of the compactor tree consist of OR gates. The output of the OR gate in the last layer (root node of the compactor tree) is connected to the photo-diode detector located in the sink of the microfluidic array.

Fig. 15 illustrates the microfluidic compactor for functional testing of 16 rows/columns. The compactor consists of one layer of microfluidic NOT gates and three layers of OR gates. In Fig. 15(a), after the two-manipulation-step merging and splitting test in the row, each of the odd electrodes has a $1 \mathrm{x}$ droplet on it, indicating that there is no malfunction in this row. According to the definitions of microfluidic logic values, we infer that the output value of the compactor is ' 0 ', indicating that there is no droplet on the electrode corresponding to the output port of the compactor. Instead of serially routing all the droplets in the row-under-test to the capacitive-sensing circuit for detection, the photo-diode detector indicates the absence of a droplet as in [11] at the output of the compactor; therefore there is no malfunction in this row.

In Fig. 15(b), the droplet on the fifth electrode undergoes a unbalanced split during functional test. Since all other droplets are split evenly, this malfunction results in a pair of test droplets of abnormal volume, one bigger and the other smaller. If the next step of test yields no malfunction, the droplet volume variation is propagated one electrode away. Note that the smaller droplet is too small (less than $0.5 \mathrm{x}$ ) to be moved into the corresponding microfluidic NOT gate, so input of this NOT gate is ' 0 '. The output of the compactor is ' 1 ', indicating the presence of a $1 \mathrm{x}$ droplet on the output port of the compactor. The photo-diode detector detects this droplet, indicating a malfunction in the row-under-test.

Fig. 16 shows the placement of the microfluidic compactor for a 16-electrode row-under-test. Note that we use simplified designs of the NOT gate consisting of eight electrodes, and the OR gate consisting of eight electrodes. The washing port and waste reservoir are not included in order to reduce the size of of the gates. The reference droplets used by the NOT and OR gates are pre-loaded into the array. Only one washing port and one waste reservoir are needed for the whole compactor. After the compaction of the test-outcome droplets, a droplet from the washing port is routed through each of the electrodes in the compactor, merged with the waste droplets, and routed to the waste reservoir. Dynamic reconfiguration is utilized to construct the microfluidic compactor, in order to use only a small amount of electrodes. In Fig. 16(a), there are two NOT gates (shown in red) in the array. At the left side of the row, four droplets are routed through the first NOT gate one-byone, while other four droplets are routed through the second NOT gate one-by-one. The eight electrodes (shown in gray) are the destinations where the outputs of the NOT gates are connected.

Using the above routing procedure, only two NOT gates are needed instead of eight NOT gates in the schematic in Fig. 15. In Fig. 16(b), the electrodes are reconfigured into two OR gates. Two pairs of droplets are routed concurrently through the OR gates. The same procedure is repeated for other two pairs, as shown in Fig. 16(c). Therefore, we only need two OR gates instead of four OR gates in the second layer of 


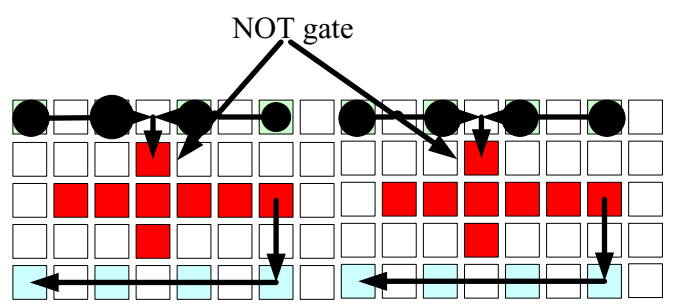

(a)

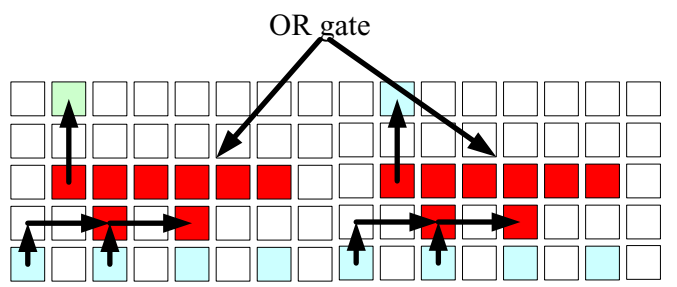

(b)

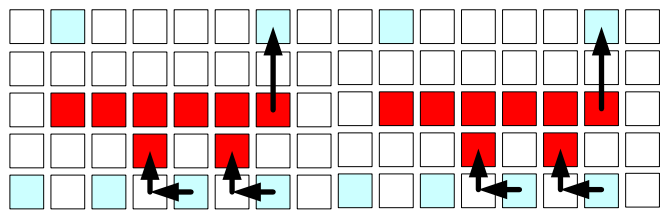

(c)

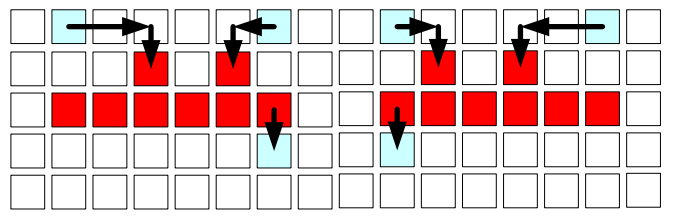

(d)

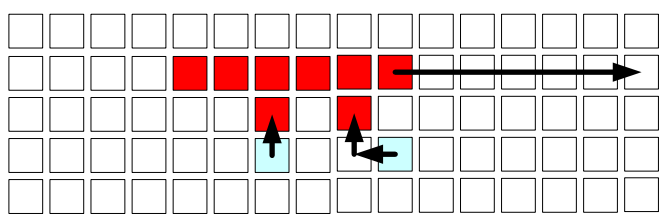

(e)

Fig. 16. Placement of the microfluidic compactor for functional testing.

1) Route $1 \mathrm{x}$ droplets to all the odd electrodes in a row;

2) Carry out splitting test for all the odd electrodes concurrently (1x droplets are now on even electrodes);

3) Carry out splitting test for all the even electrodes concurrently (1x droplets are now on odd electrodes);

4) Compress the droplets using microfluidic compactor, which is implemented by reconfiguring inverters and OR gates, then route the output of the compactor to the photodiode detector for test readout;

5) Repeat the test procedure for the next row;

6) Repeat steps 1-5 for columns.

Fig. 17. Procedure of complete mixing and splitting test procedure with reconfiguration of microfluidic compactor.

compactor. In Fig. 16(d), the electrodes are reconfigured into two OR gates to implement the function in the third layer of the compactor. In Fig. 16(e), the electrodes are reconfigured into one OR gate to implement the function in the last layer of the compactor. The complete mixing and splitting test procedure with a reconfigurable microfluidic compactor is

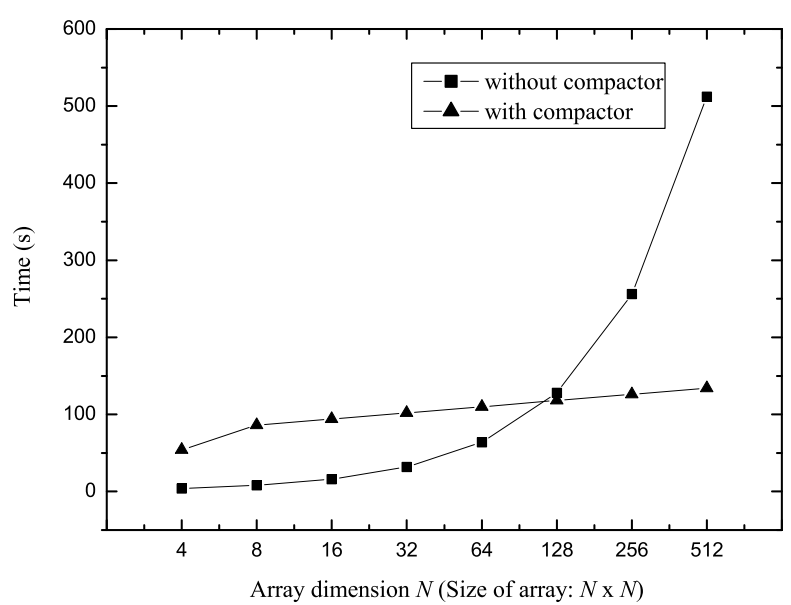

Fig. 18. Comparison of droplet-routing time with and without microfluidic compactor.

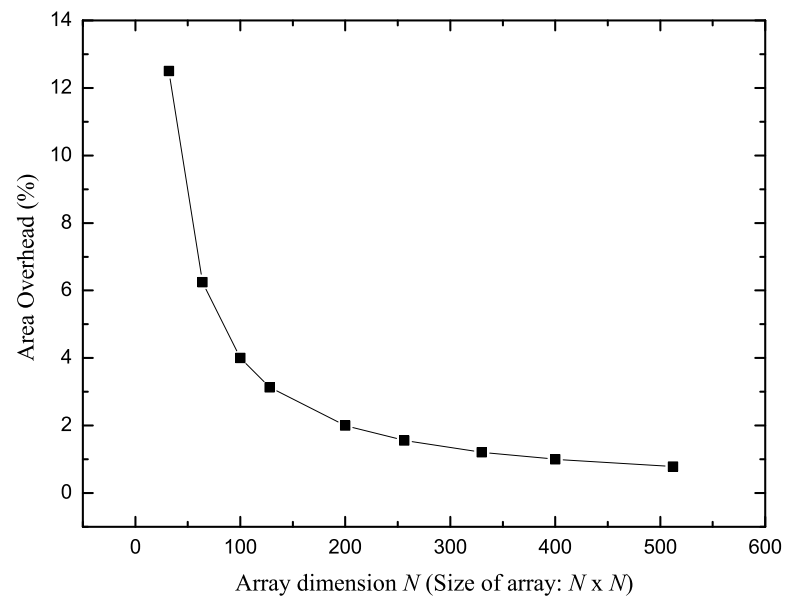

Fig. 19. Area overhead of the microfluidic compactor for functional testing. shown in Fig. 17.

For functional testing without using a compactor, an $N \times$ $N$ microfluidic array needs $N$ clock cycles to route all testoutcome droplets serially to the sink node with the capacitivesensing circuit. For functional testing with a compactor, we need $8 \times\left(\log _{2} N+4\right)$ clock cycles to compress the testoutcome droplets to one droplet. The comparison of resultevaluation time for the two methods, taking into account the 30 s needed for optical sensing and assuming a typical $1 \mathrm{~Hz}$ clock frequency, is shown in Fig. 18.

The proposed microfluidic compactor requires $4 \times N$ electrodes for an $N \times N$ array. The area overhead of the compactor as a function of $N$ is shown in Fig. 19.

\section{Application to Diagnosis of Single DEFECTIVE ElECTRODE}

In order to achieve defect tolerance via reconfiguration, diagnosis is needed to locate the faulty cells. We assume here 


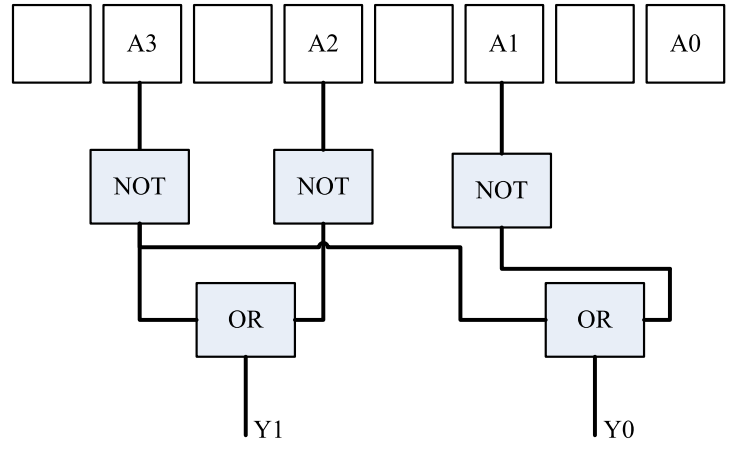

Fig. 20. Schematic of microfluidic encoder for diagnosis of a single defect in an $8 \times 8$ array.

that at most one electrode in the microfluidic array is defective. This defect can be located by analyzing the pulse-sequences of four iterations of parallel scan-like test in [17]. Such a method is prone to errors in calibration or readout.

We propose an encoder-based diagnosis method that uses the scan-like test method to identify the defective electrode. After one iteration of the parallel scan-like test, we have to check whether there is one $1 \mathrm{x}$ droplet on each of the pseudosinks. Under the assumption of a single defective cell, there is at most one pseudo-sink having no droplet. The different defect patterns can be viewed as the inputs to an encoder, which can be encoded in $\log _{2}(n+1)$ bits when the number of defect patterns is $n$. For example, in an $8 \times 8$ array, the four possible defect patterns are 1110, 1101, 1011 and 0111. Each defect pattern (A3 A2 A1 A0) can be encoded using two bits ( $\mathrm{Y} 1 \mathrm{Y} 0$ ) by a "4 to 2 encoder". If there is a defective cell on the row that uses $\mathrm{A} 2$ as its pseudo-sink, the defect pattern is (A3 A2 A1 A0) $=1011$, so the output of encoder is $\mathrm{Y} 1 \mathrm{Y0}=10$. The values of $\mathrm{Y} 1$ and $\mathrm{Y} 0$ can be read out using two photo-diode detectors. Using the above encoder-based diagnosis method, we can identify which row and column the single defect is located at by implementing four iterations (two for rows and two for columns) of parallel scan-like test.

We can use microfluidic NOT, AND and OR gates to implement the encoder. The relevant logic equations are $\mathrm{Y} 0$ $=\overline{A 3}+\overline{A 1}, \mathrm{Y} 1=\overline{A 3}+\overline{A 2}$. As shown in Fig. 20, the input ports of the microfluidic encoder are connected to the pseudosinks in the last row of the array, while each of the outputs of the encoder is connected to a photo-diode detector. For encoders with different number of inputs, the logic equations describing the corresponding truth table can be synthesized, then the microfluidic logic gates are placed to implement the encoder.

\section{Conclusions}

We have presented a new built-in self-test (BIST) method for the digital microfluidic lab-on-chip. This method utilizes digital microfluidic logic gates to implement various compactors for fault detection and diagnosis. Using the principle of electrowetting-on-dielectric, we have implemented AND, OR and NOT gates. The same input-output interpretation enables the cascading of gates to implement various logic functions. The microfluidic compactors can quickly compress the test-outcome droplets into one droplet. In this way, the proposed BIST approach obviates the need for error-prone capacitive-sensing circuits. We have also proposed a diagnosis method based on a microfluidic encoder to efficiently locate a single defective electrode in a microfluidic array. Dynamic reconfiguration is used during test and diagnosis to achieve low area overhead.

\section{ACKNOWLEDGEMENTS}

The authors thank Bang-Ning Hsu and Prof. Richard Fair for help in carrying out the laboratory experiments, and Advanced Liquid Logic, Inc. for providing prototype chips for the experiments.

\section{REFERENCES}

[1] R. B. Fair et al., "Chemical and biological applications of digitalmicrofluidic devices", IEEE Design \& Test of Computers, vol. 24, pp. 10-24, 2007.

[2] K. Chakrabarty and F. Su, Digital Microfluidic Biochips: Synthesis, Testing, and Reconfiguration Techniques, CRC Press, Boca Raton, FL, 2006.

[3] M. Tartagni and R. Guerrieri, "A fingerprint sensor based on the feedback capacitive sensing scheme", IEEE J. Solid-State Circuits, vol. 33, pp. 133-142, 1998.

[4] E. J. Griffith, S. Akella and M. K. Goldberg, "Performance characterization of a reconfigurable planar-array digital microfluidic system ", IEEE Trans. CAD, vol. 25, pp. 345-357, 2006.

[5] H. G. Kerkhoff, "Testing microelectronic biofluidic systems", IEEE Design \& Test of Computers, vol. 24, pp. 72-82, 2007.

[6] D. W. M. Marr and T. Munakata, "Micro/Nanofluidic computing", Comm. ACM, vol. 50, pp. 64-68, 2007.

[7] P. Paik, V. K. Pamula, M. G. Pollack, and K. Chakrabarty, "Coplanar Digital Microfluidics Using Standard Printed Circuit Board Processes," MicroTAS, 2005.

[8] M. Prakash and N. Gershenfeld, "Microfluidic bubble logic", Science, vol. 315, pp. 832-835, 2007.

[9] A. J. Ricketts, K. Irick, N. Vijaykrishnan, and M. J. Irwin, "Priority scheduling in digital microfluidics-based biochips", Proc. DATE Conf., pp. 329-334, 2006.

[10] Silicon Biosystems. www.siliconbiosystems.com

[11] V. Srinivasan, V. K. Pamula, M. G. Pollack and R. B. Fair, "Clinical diagnositics on human whole blood, plasma, serum, urine, saliva, sweat, and tears on a digital microfluidic platform", Proc. MicroTAS, pp. 12871290, 2003.

[12] F. Su, W. Hwang, A. Mukherjee and K. Chakrabarty, "Testing and diagnosis of realistic defects in digital microfluidic biochips", JETTA, vol. 23, pp. 219-233, 2007.

[13] F. Su, K. Chakrabarty and R. B. Fair, "Microfluidics-based biochips: technology issues, implementation platforms, and design automation challenges", IEEE Trans. CAD, vol. 25, pp. 211-223, 2006.

[14] T. Vestad et al., "Flow resistance for microfluidic logic operations", Applied Physics Letters, vol. 84, no. 25, pp. 5074-5075, 2004.

[15] P. H. Yuh, C. L. Yang, and C. W. Chang, "Placement of defect-tolerant digital microfluidic biochips using the T-tree formulation", ACM J. Emerging Tech. Computing Sys., vol. 3, pp. 13.1-13.32, 2007.

[16] W. Zhan and R. M. Crooks, "Microelectrochemical logic circuits", J. Am. Chem. Soc., vol. 125, no. 33, pp. 9934-9935, 2003.

[17] T. Xu and K. Chakrabarty, "Parallel scan-like test and multiple-defect diagnosis for digital microfluidic biochips", IEEE Trans. Biomedical Circuits and Sys., vol. 1, pp. 148-158, 2007.

[18] T. Xu and K. Chakrabarty, "Functional testing of digital microfluidic biochips", Proc. ITC, 2007.

[19] T. Xu, P. Thwar, V. Srinivasan, V. K. Pamula, and K. Chakrabarty, "Digital microfluidic biochip for protein crystallization", IEEE-NIH Life Science Systems and Applications Workshop, Bethesda, MD, 2007. 